\title{
Inovações tecnológicas em genética bovina e maturidade gerencial dos pecuaristas
}

\section{Nilton Cesar Lima}

Doutor em Administração pela Universidade de São Paulo. Professor do Programa de Pós graduação em Ciências Contábeis da Universidade Federal de Uberlância

niltoncesar@ufu.br

Gustavo Henrique Silva de Souza

Mestre em Psicologia pela Universidade Federal de Alagoas . Professor no Instituto Federal de Educação Ciência e Tecnologia do Norte de Minas Gerais

souza.g.h.s@gmail.com

Jorge Artur Peçanha de Miranda Coelho

Doutor em Psicologia Social pela Universidade Federal da Paraíba. Atualmente é Professor Adjunto da Universidade Federal de Alagoas

jorge.coelho@famed.ufal.br

José Eduardo de Aguiar

Doutor em Saneamento, Meio Ambiente e Recursos Hídricos pela Escola de Engenharia da UFMG

Professor da Universidade Federal de Uberlândia

guiar@ufu.br

\section{Editor Científico: José Edson Lara}

Organização Comitê Científico

Double Blind Review pelo SEER/OJS

Recebido em 23.06.2018

Aprovado em 15.11.2018

Este trabalho foi licenciado com uma Licença Creative Commons - Atribuição - Não Comercial 3.0 Brasil 

pecuaristas

\title{
Resumo
}

O presente artigo teve por objetivo identificar o grau de maturidade em gestão tecnológica das empresas pecuaristas brasileiras usuárias de técnicas de genética bovina e levantar os elementos principais que balizam tal constructo. Para tanto, foi realizado um estudo com 1.436 empresas pecuaristas. Os resultados mostraram que as empresas pecuaristas investigadas estão em um grau médio (intermediário) de maturidade em gestão tecnológica, representando $74,7 \%$ da amostra. Além disso, a maturidade em gestão tecnológica foi explicada (1) pela adoção de práticas de mensuração da produtividade e de ganhos econômicos em relação à gestão do negócio, (2) pela rentabilidade associada ao emprego da tecnologia em sexagem genética, (3) pelos conhecimentos sobre a rentabilidade do negócio e (4) pela adoção de ferramentas robustas de gestão de custos - especificamente o COE e o COT. Basicamente, os resultados são contundentes em indicar que as empresas pecuaristas brasileiras são profícuas no uso das inovações tecnológicas em genética bovina.

Palavras-chave: Gestão Tecnológica; Maturidade Gerencial; Genética Bovina; Empresas Pecuaristas.

\section{Technological innovations in bovine genetics and management maturity of pecuarists}

\begin{abstract}
This article aimed to identify the degree of maturity in technological management of Brazilian enterprises ranchers, users of bovine genetics techniques, and lift the main elements which guide this construct. To this end, a study was conducted with 1,436 ranchers. Results showed that enterprises ranchers are in a medium degree of maturity in technological management, accounting for $74.7 \%$ of the sample. In addition, the maturity in technological management was explained (1) by adopting measurement practices of productivity and economic gains regarding to business management, (2) by the profitability associated with the use of genetic sexing technology, (3) by the knowledge about the profitability of the business and (4) by the adoption of robust tools of cost management - specifically the COE and the COT. Basically, results are conclusive to indicate that Brazilian enterprises ranchers are fruitful in the use of technological innovations in bovine genetics.
\end{abstract}

Keywords: Technological Management; Managerial Maturity; Bovine Genetics; Ranchers.

\section{Innovaciones tecnológicas en genética bovina y madurez gerencial de los ganaderos}

\section{Resumen}

El presente artículo tuvo por objetivo identificar el grado de madurez en gestión tecnológica de las empresas pecuaristas brasileñas usuarias de técnicas de genética bovina y levantar los elementos principales que balizan tal constructo. Para ello, se realizó un estudio con 1.436 empresas ganaderas. Los resultados mostraron que las empresas ganaderas investigadas están en un grado medio (intermedio) de madurez en gestión tecnológica, representando el 74,7\% de la muestra. Además, la madurez en gestión de la tecnología se explica (1) la adopción de prácticas de medición de la productividad y beneficios económicos en relación con la gestión de negocios, (2) la rentabilidad asociado con el uso de la tecnología en sexaje genético, (3) por conocimientos sobre la rentabilidad del negocio y (4) por la adopción de herramientas robustas de gestión de costos - específicamente el COE y el COT. Básicamente, los resultados son 
contundentes en indicar que las empresas ganaderas brasileñas son profícuas en el uso de las innovaciones tecnológicas en genética bovina.

Palabras clave: Gestión Tecnológica; Madurez Gerencial; Genética Bovina; Empresas Ganaderos.

\section{Introdução}

Pesquisas em genética animal têm sido desenvolvidas em todo o mundo sobre a tecnologia da sexagem de espermatozoides - capaz de separar os cromossomas X e Y -, para determinar o sexo do animal a ser gerado (Checa et al., 2002; Garner, 2006; Baruselli et al., 2007; Freitas, 2007; Seidel Jr., 2007; Sharpe \& Evans, 2009; Alkmin et al., 2014). Para a pecuária bovina no Brasil, em especial, a técnica já representa uma realidade aplicada e comercializada dentre as empresas de tecnologia genética (Beltrame et al., 2010). A técnica tem como princípio a Citometria de Fluxo, responsável por variadas análises simultâneas de partículas microscópicas, os quais são detectados os cromossomos em um fluxo de fluído, definindo minuciosamente as características físico-químicas da célula, ao passar por um aparelho de detecção óptico-eletrônico (Gao et al., 2009; Sharpe \& Evans, 2009).

Essa inovação tecnológica em genética bovina associada a outras já existentes, como a inseminação artificial e a fertilização in vitro, acelera o ganho genético do animal, além de ser capaz de definir proficuamente o sexo do bezerro (Trigal et al., 2012). Em termos de produção em escala, o uso da inseminação artificial otimiza a capacidade produtiva e amplia a possibilidade de maiores ganhos financeiros e rentabilidade, tanto para o produtor de gado de leite - que necessita obter um rebanho estritamente de fêmeas -, quanto para o pecuarista produtor de gado de corte - que necessita obter um rebanho estritamente de machos, por esse apresentar ganho de peso mais precoce (Lima, 2007; Varago et al., 2008; Beltrame et al., 2010).

Isto significa que, com a viabilidade comercial para a tecnologia pecuária, representando um grande diferencial para o segmento, os resultados financeiros de empresas do ramo da pecuária bovina tenderão a crescer expressivamente, segundo Beltrame et al. (2010). Para os autores, já há uma expectativa da inovação em sexagem de espermatozoides para a cadeia produtiva da pecuária bovina, com as seguintes características positivas: (1) não alteração da viabilidade do uso do esperma; (2) compatibilidade com a congelação do espermatozoide sexado; (3) possibilidade de sexagem de espermatozoides previamente congelados e descongelados; e, (4) produção de várias doses de sêmen sexado congelado por dia, com custo compatível ao mercado. 

pecuaristas

De tal modo, a técnica da sexagem genética e da fertilização in vitro (visando o melhoramento genético do animal) conjuntamente associada à sua viabilidade econômica, estimula toda a cadeia produtiva da pecuária bovina à maior produtividade, competitividade $\mathrm{e}$ aprendizagem. Surge, então, a necessidade de investigar como as inovações tecnológicas em genética bovina em seu estágio atual vêm se caracterizando como evidentes vantagens mercadológicas. Assim, o objetivo deste artigo é identificar o grau de maturidade em gestão tecnológica das empresas pecuaristas brasileiras usuárias de técnicas de genética bovina e levantar os elementos principais que balizam tal constructo.

O presente estudo baseia-se, particularmente, em inovações tecnológicas nos setores operacionais da organização, que afetam a produtividade e a competitividade pela otimização dos custos e da qualidade, concebendo estes como os principais efeitos de ondulação positiva na maturidade gerencial das empresas (Harmon, 2004; Nascimento et al., 2013; Nascimento et al., 2014).

\section{Fundamentação Teórica}

A pertinência de uma fundamentação teórica, como ressalta Whetten (2003), está em construir uma estruturação conceitual que dê sustentação às proposições de pesquisa de cada estudo em particular. Nesse sentido, este estudo é específico em três temas centrais, discutidos nas próximas seções: (1) Inovações Tecnológicas; (2) Inovações em Genética Bovina no Mercado Pecuarista; e (3) Maturidade Gerencial.

\section{Inovações Tecnológicas}

Há vários estudos acerca do gerenciamento de inovações tecnológicas. Grande parte desses estudos está enraizada em dois ramos distintos de economia aplicada: o comércio associado aos níveis de investimentos (Judice \& Baêta, 2005; Ramos \& Zilber, 2015; Burrus et al., 2018; Kostis et al., 2018) e as capacidades tecnológicas em inovação produtiva (MatiasPereira \& Kruglianskas, 2005; Huang \& Chen, 2010; Wignaraja, 2012; Parolin, 2013, Roud, 2018).

Especificamente, sobre a implantação de inovações tecnológicas, Clark \& Fujimoto (1991) explicam que a forma mais comumente utilizada durante o século XX foi a organização e o gerenciamento da produção apenas pelo desenvolvimento de novos produtos. No entanto, nos contextos da globalização e devido ao foco nas demandas criadas pelos próprios consumidores, as formas de organizar a produção passaram a emergir da externalização de 
atividades para os fornecedores (outsourcing), tendo-se a cadeia produtiva como elemento estratégico.

Inovação operacional envolve, portanto, uma decisão importante para a empresa: saber quando externalizar atividades inerentes ao processo de desenvolvimento tecnológico - em sobrepujo à internalização - e como coordenar as atividades entre fornecedores e indústrias no desenvolvimento de produtos. Para Fine \& Whitney (1999), este é um processo claro de terceirização de competências essenciais, podendo ocorrer por duas razões estruturais: (1) dependência de capacidade, quando uma empresa decide externalizar uma atividade por motivos econômicos ou outros diversos, mas possui o conhecimento necessário para executála, caso fosse necessário ou desejável fazê-lo; ou (2) dependência de conhecimento, quando uma empresa não tem a competência necessária para desenvolver ou produzir determinados componentes-chave para o seu produto e depende de terceiros para supri-lo. Sabe-se que a evolução tecnológica tem em si a aprendizagem, cujas regras do modelo de decisão permitem que inovações radicais ocorram para o mercado, gerando padrões de difusão tecnológica a serem observadas no mundo real (Miguel et al., 2013; Penedo et al., 2014; Roud, 2018). Em contraponto, a perspectiva adotada por Krishnan \& Ulrich (2001) avalia que a superioridade e a credibilidade tecnológica trafegam por um conceito de desenvolvimento associado a formas criativas e a configurações dentro das esferas do marketing, da engenharia, da gestão e das operações. Ou seja, o desenvolvimento não está no produto operacionalizado e pronto para comercialização, mas sim, na capacidade criativa de pessoas que identificam a necessidade e a aderência do produto a ser desenvolvido.

Em paralelo, Hill \& Rothaermel (2003) percebem este fator como o ponto em que as tomadas de decisão são vistas pela avaliação dos investimentos em tecnologia e sua respectiva perspectiva de realização. Para os autores, o período de gestação que demarca a maturação tecnológica está associado à dimensão histórica do desempenho da empresa na concretização de um novo produto. Em concordância, Srinivasan et al., (2006) afirmam que a tecnologia dominante tem suas características ligadas ao tempo, responsável pelo: surgimento condicional da tecnologia, características do mercado, rentabilidade associada ao produto, processo de definição de normas e inovação radical.

Huang \& Chen (2010) sugerem que as empresas não devem ser muito especializadas, nem muito diversificadas na estratégia de desenvolvimento de tecnologia, uma vez que a homogeneidade não permite alcançar um desempenho melhor em inovação. É, portanto, 

pecuaristas

necessária a conciliação entre a economia de escopo e o compartilhamento de conhecimento, sendo estes responsáveis pelo desempenho da inovação. De tal modo, empresas com diversidade tecnológica podem utilizar-se de diferentes recursos organizacionais a fim de melhorar resultados em inovação. Isso significa, segundo Suarez (2004), que o lançamento de um novo produto sem ter uma base definitiva instalada leva a custos mais elevados para os clientes. Além disso, conforme Hill \& Rothaermel (2003) destacam, a comercialização da inovação é altamente beneficiada por ativos complementares, como a diversidade tecnológica.

Wignaraja (2012) corrobora essa linha de pensamento. Para o autor, a inovação e o aprendizado representam o principal determinante da capacidade competitiva da empresa. Sob o ponto de vista da motivação pela inovação tecnológica, Pellegrino et al. (2012) argumentam que a inovação não deve ser o foco principal de uma organização, mas sim, o desempenho em termos de produtividade e rentabilidade associada a níveis mais robustos de inovação. O que, na perspectiva adotada por Camargo et al. (2004) e Camargo et al. (2006), caracteriza o mercado pecuarista atual em relação às práticas inovadoras em genética bovina.

\section{Inovações em Genética Bovina no Mercado Pecuarista}

Especificamente no ramo pecuarista, as inovações tecnológicas caracterizam-se em duas categorias distintas: (1) Tecnologias da Informação e Comunicação (TICs) e (2) Biotecnologias - correspondentes ao melhoramento genético ou à biologia molecular (Rege et al., 2011). Neste estudo, iremos abordar as tecnologias associadas à biologia molecular, no que compete às ferramentas genômicas, que oferecem possíveis avanços na formação genética e no melhoramento da raça bovina.

Rege \& Gibson (2003) explicam que, dentre as novas tecnologias para o melhoramento na produção pecuária de bovinos, particularmente as tecnologias associadas à mudança genética, como a sexagem genética e a fecundação in vitro, oferecem grandes perspectivas inovadoras ao mercado produtor e à cadeia produtiva.

\section{Sexagem Genética}

De acordo com Baruselli et al. (2007), a sexagem genética caracteriza-se pela separação dos espermatozoides "machos" (cromossomo Y) dos espermatozoides "fêmeas" (cromossomo $\mathrm{X}$, que possui cerca de $4 \%$ a mais de material genético). A técnica é realizada a partir de um equipamento a laser (citômetro de fluxo) que emite feixes de luz ultrasensitivos, promovendo a fluorescência das células espermáticas previamente coradas, o que permite a separação dos 
espermatozoides X e Y com membrana celular intacta - uma tecnologia difundida pela empresa Sexing Technologies (2016).

Segundo a Sexing Technologies (2016), a técnica tem demonstrado que o gênero do animal tem sua comprovação média em $93 \%$ dos casos, desde que o protocolo de uso seja aplicado adequadamente em novilhas virgens e em bom estado de nutrição e ciclo reprodutivo.

Seidel Jr. (2007) destaca que, em programas de melhoramento genético, os bezerros nascidos de sêmen sexado são geneticamente superiores. Apesar disso, o fator econômico continua sendo o principal impulsionador da implementação da tecnologia de sêmen sexado (Seidel Jr., 2003; Freitas, 2007; Pellegrino, et al., 2016; Hall et al., 2017). Basicamente, segundo Camargo et al. (2006), as empresas pecuaristas estão investindo na sexagem genética, visando maior competitividade e diferenciação no oferecimento de produtos geneticamente melhorados associados a ganhos econômicos. Portanto, em termos de produtividade, ressaltase a possibilidade de produzir mais com menores custos.

No entanto, o sistema de sexagem não é operacionalmente eficiente (Garner, 2006), pois a produção do sêmen sexado, desde a coleta do sêmen até o envasamento, leva em torno de 9 minutos - tempo acima do necessário para o suprimento de um processo produtivo normal (inseminação com doses de sêmen na concentração convencional). Isto é, a tecnologia da sexagem genética, comparada ao processo de inseminação convencional, impõe um ritmo menor de produtividade, uma vez que o tempo de separação dos cromossomos na máquina de citometria de fluxo onera o processo produtivo (Seidel Jr., 2007).

Garner \& Seidel Jr. (2008) e Seidel Jr. (2007, 2009) explicam, por outro lado, que isso não inviabiliza o sistema de sexagem. Para tanto, existem duas maneiras para lidar com este problema, ambas envolvendo a utilização de poucos espermatozoides por dose. De tal modo, a primeira solução consiste na seleção de reprodutores que, com baixas concentrações do sêmen apresentam uma boa fertilidade; e a segunda solução, é a mudança no protocolo de aplicação do sêmen sexado, orientando o uso da técnica em vacas com boas condições reprodutivas e de manejo.

Sob o ponto de vista econômico, Baruselli et al. (2007) ressalta a importância da tecnologia quanto à sua usabilidade na maximização da produção animal, uma vez que na bovinocultura o sexo do bezerro é um dos fatores determinantes para o desempenho do agronegócio. O desafio, na verdade, está na plena aplicação ou difusão da técnica dentre o segmento pecuário, visto que a técnica de sêmen sexado ainda é limitada devido às baixas taxas 

pecuaristas

de prenhez (decorrentes do emprego indevido do protocolo de utilização), ao tempo da sexagem pelo citômetro de fluxo, à baixa concentração de espermatozoide por dose na inseminação, ao alto custo da dose ao pecuarista e à complexidade de armazenamento de espermatozoides.

Assim, um dos paradigmas enfrentados pelas indústrias da inseminação artificial é dificuldade da produção em escala comercial de doses de sêmen sexado, visando maior acessibilidade e aplicação pelos pequenos pecuaristas. Camargo et al. (2006) explica que a aprendizagem organizacional pelo desenvolvimento da técnica, no sentido de aperfeiçoamento para que o uso em larga escala seja viável, é efetiva para os pecuaristas de um modo geral. Segundo os autores, grande parte dos produtores pecuaristas tem associado a técnica da sexagem genética à fecundação in vitro, o que, teoricamente, seria uma solução para os problemas iniciais da produção em escala.

\section{Fertilização in vitro}

Nos últimos anos, segundo Trigal et al. (2012), a técnica de Fertilização in vitro (FIV) teve avanços consideráveis no mercado pecuarista. Associada à maturação in vitro de oocistos, a FIV tem sido utilizada para produção de grande número de embriões de fêmeas de várias idades e estados fisiológicos, que são usados para pesquisa ou para produção comercial. Varago et al., (2008) destacam que a FIV é uma técnica fundamental para o uso das novas biotécnicas de reprodução animal, em função da possibilidade de produzir embriões pró-sexados e embriões ou zigotos em vários estágios de desenvolvimento, para os estudos de transgênese e clonagem. Ademais, a FIV vem sendo utilizada para avaliar o potencial reprodutivo de touros, indicando com mais precisão a fertilidade de um reprodutor.

Segundo a Empresa Brasileira de Pesquisa Agropecuária (Embrapa, 2018), no sentido literal, a fertilização in vitro (FIV) se refere à interação de espermatozoide-oocisto in vitro, com a formação de um novo indivíduo. Em sentido amplo, corresponde à combinação de processos necessários à produção de embriões em laboratório. Nesse caso, envolve a maturação de oocistos imaturos aspirados dos ovários, a capacitação espermática, a fecundação dos oocistos e o cultivo de embriões até o estágio de blastocisto - quando estão prontos para serem transferidos para as fêmeas receptoras (Cavalieri et al, 2018).

Não obstante, a FIV apresenta limitações operacionais que dificultam a preservação dos embriões produzidos por essa técnica, consequentemente, maiores custos com embriões. De acordo com a Embrapa (2018), a técnica necessita ser aperfeiçoada, no sentido de elevar os índices de produção e, assim, reduzir os custos. Para Varago et al., (2008), o desenvolvimento 
de uma técnica de criopreservação adequada para a produção in vitro de embriões é a principal forma de possibilitar a utilização maximizada da tecnologia de produção embrionária in vitro, dentro de uma economia de escala.

Beltrame et al. (2010) corroboram essa linha de pensamento. Para os autores, a técnica de produção in vitro de embriões bovinos depende de uma sincronização do número de vacas receptoras e de uma adaptação dos protocolos de utilização, para que haja uma significativa diminuição dos custos finais da técnica. Basicamente, o uso do número ótimo de receptoras por doadora proporciona uma produção com menor custo. Assim, para o atendimento de uma larga escala comercial dentre os pecuaristas, a técnica exigiria conhecimento na aplicação e métodos de aplicação condizentes ao protocolo de utilização - representando um desafio proeminente para o mercado pecuarista.

\section{Implicações Mercadológicas}

Conforme Camargo et al. (2006) esclarece, a viabilidade de inovações tecnológicas em genética bovina para empresários pecuaristas não se resume à técnica em si, mas constringe aspectos econômicos e mercadológicos, no que concerne à maior rentabilidade para a cadeia produtiva em ganhos de escala, diferenciação competitiva e maior grau de aprendizagem.

Especificamente, em relação aos ganhos de escala para empresas pecuaristas e, consequentemente, à elevada produtividade, a Associação Brasileira de Inseminação Artificial (ASBIA, 2018) explica que um touro (progenitor) em regime de cruzamento controlado tem um índice de reprodução (respondendo a cerca de 30 vacas) de 120 a 400 bezerros, considerando 4 anos de vida útil. Por outro lado, com a inseminação associada à sexagem genética, um touro (progenitor) em regime de cruzamento controlado tem um índice de reprodução de 100.000 bezerros, considerando o mesmo período de tempo.

Isso ocorre, porque a técnica possibilita ganho de peso, precocidade, rusticidade e musculatura para o touro que, geneticamente, podem ser transferidas para outros animais (bezerros), tornando-se uma diferenciação em produtividade. Beltrame et al. (2010) considera que essa inovação tecnológica tenderá a fortalecer, cada vez mais, a cadeia produtiva pecuarista, especialmente, devido à adoção da sexagem genética, que permite maior ganho de escala pelo pecuarista.

De tal modo, o enfoque parte, precisamente, da tecnologia em sexagem genética, tendo como segundo plano o emprego da fecundação in vitro, em que ambos possibilitam a aceleração da formação de novos progenitores, permitindo caracterizar vantagem produtiva, competitiva e 

pecuaristas

aprendizagem em inovações ao segmento (Camargo et al., 2006; Varago et al., 2008; Beltrame et al., 2010). Para Camargo et al. (2006), essa alavancagem produtiva e competitiva em função do uso de inovações tecnológicas é efeito de um processo de internalização e aprendizagem organizacional que acarreta em graus mais elevados de maturidade gerencial das empresas pecuaristas.

\section{Maturidade Gerencial}

A maturidade gerencial é um constructo que, comumente, é atribuído à instituição de práticas de planejamento e controle que convergem em processos sistemáticos para a obtenção de eficácia organizacional. De modo geral, os modelos de maturidade gerencial estabelecem níveis que avaliam o desempenho organizacional associado à melhoria de práticas de gerenciamento (Harmon, 2004).

Nascimento et al. (2014) investigaram dimensões determinantes para a maturidade gerencial. Para os autores, a maturidade está na conjunção de elementos, como competência técnica, uso de metodologia, estrutura organizacional e alinhamento com o negócio (estratégia). Isto é, engloba o uso consistente de uma cadeia de ferramentas, habilidades, conhecimentos, técnicas e processos em função de objetivos predefinidos.

Os modelos de maturidade se focam na atribuição de indicadores que comparam desempenho gerencial. De tal modo, a maturidade diz respeito a altos níveis de sofisticação no uso de técnicas de gerenciamento (Nascimento et al., 2013; Nascimento et al., 2014), o que inclui, em consonância a Harmon (2004), o efeito da inovação tecnológica nos setores operacionais e produtivos da organização.

De tal modo, índices de gerenciamento construídos com base na atividade tecnológica profícua (produtividade e competitividade) e na configuração da otimização dos custos e da qualidade podem indicar, portanto, níveis de maturidade em gestão tecnológica (Harmon, 2004) - explicada por uma probabilidade de sucesso ou de êxito associada.

O embasamento teórico que dá sustentação a este pressuposto é o mesmo utilizado por Harmon (2004) - na mensuração de graus de gestão - ou por Nascimento et al. (2014) - na mensuração da maturidade gerencial em projetos. Tem-se a maturidade, então, como um processo em que a organização adquire competências elevadas, gradativamente, em consequência à aquisição de posicionamentos estratégicos (planejamento), ao estabelecimento de padrões gerenciais (condução), à instituição de parcerias dentro do mercado (integração) e à adoção de práticas inovadoras (aperfeiçoamento). 
Em função dessa perspectiva de análise, o presente estudo fundamenta-se na mensuração da maturidade em gestão tecnológica, convergindo com as idiossincrasias do setor pecuário e das empresas investigadas, conforme apresentar-se-á detalhadamente na próxima seção.

\section{Métodos}

\section{Tipo de Pesquisa}

Em termos metodológicos, este estudo se delineou em formato teórico-empírico, de caráter descritivo na maneira de conduzir a pesquisa e de abordagem quantitativa no modo de análise, cujo objetivo foi identificar o grau de maturidade em gestão tecnológica das empresas pecuaristas brasileiras usuárias de técnicas de genética bovina e levantar os elementos principais que explicam o constructo avaliado (a maturidade gerencial). A pesquisa descritiva associada a modelos quantitativos de análise, segundo Malhotra (2011), é traçada pelo estabelecimento de relações entre variáveis de uma amostra e determina sua relação com os fenômenos circundantes, descrevendo-os para proporcionar uma visão específica do problema.

\section{Procedimentos e Instrumentalização}

Para a realização do estudo, foi necessário o desenvolvimento de um modelo profícuo de mensuração da maturidade em gestão tecnológica, que demonstrasse funcionalidade e eficácia preditiva. Para tanto, adequando-se os pressupostos da maturidade gerencial (ver seção 2.3) ao gerenciamento das empresas pecuaristas usuárias de técnicas de genética bovina, foram elaboradas 9 questões que buscaram investigar junto às empresas:

- Uso de inseminação artificial (Baruselli et al., 2007);

- Tempo de uso de técnicas de genética bovina (Beltrame et al., 2010; NASCIMENTO et al., 2014);

- Conhecimentos sobre rentabilidade (Camargo et al., 2004);

- Rentabilidade associada à técnica de Inseminação artificial (Camargo et al., 2006).

- Rentabilidade associada à gestão inovadora do negócio (Alvarenga et al., 2014);

- Uso do COE (Custo Operacional Efetivo) e do COT (Custo Operacional Total) como ferramentas de gestão (Alvarenga et al., 2014);

- Uso extensivo de parcerias estratégicas dentro da cadeia produtiva (Julca-Briceño \& Neves, 2011); 

pecuaristas

- Mensuração da produtividade e dos ganhos econômicos associados à técnica de inseminação artificial (Rege \& Gibson, 2003; Beltrame et al., 2010).

- Mensuração da produtividade e dos ganhos econômicos associados à gestão do negócio (Bylund \& McCaffrey, 2018).

Por conseguinte, as questões foram transformadas em itens, sem modificação semântica e de conteúdo - considerando critérios normativos de instrumentalização (Hair et al., 2010; Cohen et al., 2014) - de modo que as respostas fossem interpretadas sob a forma de índice, em uma escala do tipo Likert e somada de 3 pontos, visando medir o grau de maturidade em gestão tecnológica (Baixo, Médio e Alto).

Considerando que cada um dos 9 itens podem variar em uma escala de 3 pontos, cada empresa investigada pode ter escore somado no intervalo de 9 (mínimo) a 27 (máximo) pontos totais, que indicam em que grau de maturidade em gestão tecnológica cada uma está. Assim, utilizando uma divisão ponderada simples, ajuizou-se uma interpretação às pontuações dentro de intervalos do escore, conforme o quadro 1:

\begin{tabular}{|c|c|l|}
\hline $\begin{array}{c}\text { Intervalo } \\
\text { do } \\
\text { Escore }\end{array}$ & Grau & \multicolumn{1}{c|}{ Interpretação } \\
\hline $\mathbf{9 - 1 5}$ & Baixo & $\begin{array}{l}\text { A empresa não dá uma concepção gerencial ao uso da inseminação artificial } \\
\text { associada à sexagem genética. O uso da tecnologia existe, entretanto, a empresa } \\
\text { provavelmente possui problemas, como a falta de conhecimento sobre } \\
\text { rentabilidade ou controle gerencial (custos e produtividade). Tempo de uso } \\
\text { incipiente das técnicas de genética bovina ou a não utilização do COE e do COT } \\
\text { são fatores que podem acarretar em baixo grau de maturidade em gestão } \\
\text { tecnológica. }\end{array}$ \\
\hline $\mathbf{1 6 - 2 0}$ & Médio & $\begin{array}{l}\text { A empresa dá uma concepção gerencial ao uso da inseminação artificial associada } \\
\text { à sexagem genética. A empresa possui conhecimentos sobre rentabilidade e } \\
\text { reconhece a contribuição da tecnologia ou da gestão inovadora à rentabilidade, em } \\
\text { geral, devido às práticas de mensuração da produtividade e dos ganhos } \\
\text { econômicos e às parcerias estratégicas. A não utilização do COE e do COT é um } \\
\text { fator que podem diminuir o grau de maturidade em gestão tecnológica. }\end{array}$ \\
\hline $\mathbf{2 1 ~ - ~ 2 7}$ & Alto & $\begin{array}{l}\text { A empresa está totalmente alinhada ao uso da inseminação artificial associada à } \\
\text { sexagem genética. O uso da tecnologia converge com padrões de gerenciamento } \\
\text { inovadores, atribuído às práticas de mensuração da produtividade e dos ganhos } \\
\text { econômicos e às parcerias estratégicas. Em geral, são empresas que já utilizam } \\
\text { técnicas de genética bovina há muito tempo e fazem o uso do COE e do COT } \\
\text { como ferramentas de gestão. }\end{array}$ \\
\hline
\end{tabular}

Quadro 1: Interpretação do Grau de Maturidade em Gestão Tecnológica Fonte: Desenvolvido pelos autores.

Embora a mensuração do grau de maturidade em gestão tecnológica possua um viés subjetivo - assim como qualquer medida associada à maturidade (Harmon, 2004) -, do ponto de vista teórico, a sua instrumentalização seguiu um viés funcionalista. Basicamente, a interpretação pode ser adaptada a outros contextos organizacionais. Neste estudo, a 
interpretação dada foi estabelecida em função de uma necessidade de operacionalização dos resultados da pesquisa.

Complementarmente, foi acrescido um item ao instrumento que visou unicamente identificar em que nível os gestores atribuíam relação ao aumento da produtividade em função da tecnologia genética. Para tanto, utilizou-se o item em uma escala do tipo likert de 5 pontos, variando de $1=$ Muito Baixo à $5=$ Muito Alto. $\mathrm{O}$ intuito foi conhecer a opinião pessoal do gestor responsável quanto à tecnologia genética, independente da adoção de práticas de mensuração de produtividade e rentabilidade ou de controle de custos.

\section{Amostra e Coleta de Dados}

Participaram do estudo 1.436 empresas pecuaristas brasileiras usuárias de técnicas de genética bovina - com o tempo médio de uso da técnica de 14,57 anos (DP $=4,159)$. As empresas participantes foram oriundas de 11 Estados Federativos do Brasil, sendo os Estados com maior incidência: São Paulo (22,9\%), Minas Gerais (15\%), Mato Grosso do Sul (14,8\%), Mato Grosso $(11,4 \%)$ e Goiás $(11,2 \%)$.

O instrumento de pesquisa foi aplicado de forma não probabilística, por acessibilidade e de forma individual, junto aos gerentes de produção ou responsáveis pelo setor produtivo de cada uma das empresas investigadas. Assim, os participantes da pesquisa foram abordados pessoalmente em seus ambientes de trabalho e esclarecidos sobre o anonimato e sigilo de suas respostas, sendo garantido o caráter voluntário da participação, bem como, o respeito às diretrizes éticas que regem a pesquisa com seres humanos. Utilizou-se o método survey, com a estratificação considerada a 95\% de grau de confiança, com margem de erro amostral padrão de $\pm 5 \%$.

\section{Análises}

Os dados foram processados no software Statistical Package for Social Sciences (IBM/SPSS 21). Inicialmente, procedeu-se às análises de estatística descritiva de parametrização simples (frequência, percentual, média e desvio-padrão). As análises iniciais foram utilizadas para identificar e apresentar o grau de maturidade em gestão tecnológica, em consonância ao objetivo proposto.

Por conseguinte, visando conhecer os elementos que melhor explicam a maturidade em gestão tecnológica (considerando apenas os 9 itens utilizados na escala de 3 pontos), ajuizouse o uso da análise fatorial de componentes principais - para fins de redução de itens -, com o 
objetivo de encontrar o conjunto mais parcimonioso de elementos que explicam o constructo avaliado (Hair et al., 2010). Verificada a adequação dos dados e a fatoriabilidade da matriz de correlações dos itens, por meio das estatísticas de Kaiser-Meyer-Olkin $(\mathrm{KMO})=0,609$ e do Teste de Esfericidade de Bartlett, $\chi^{2}(36)=1983,549 ; p=0,000$, procedeu-se à análise fatorial fixada em 1 fator (fator latente a ser reduzido), com matriz não-rotacionada. Para garantir significância prática dos itens selecionados, procedimentos mais rigorosos foram necessários, o que exigiu, segundo recomendações de Hair et al. (2010), utilizar um valor igual ou superior a carga fatorial de $|0,50|$.

Em complemento, utilizou-se a Correlação $r$ de Pearson (2 extremidades - bicaudal) e o Coeficiente de Associação $\left[\chi^{2}(1) \geq 3,84 ; p<0,05\right]$ para dar suporte à interpretação dos resultados. Utilizou-se como base analítica a evidenciação empírica indutiva, decorrente do formato como os dados foram tratados e como os resultados foram interpretados à luz do objetivo proposto (Hair et al., 2010).

\section{Resultados e Discussão}

\section{Grau de Maturidade em Gestão Tecnológica em Empresas Pecuaristas Brasileiras}

Os resultados mostram que as empresas pecuaristas brasileiras fazem parte de um grupo bastante homogêneo, no que diz respeito ao grau de maturidade em gestão tecnológica. As empresas pecuaristas investigadas mostraram-se em um grau médio (intermediário) de maturidade em gestão tecnológica, representando 74,7\% da amostra (1.073 empresas), conforme a Figura 1. 


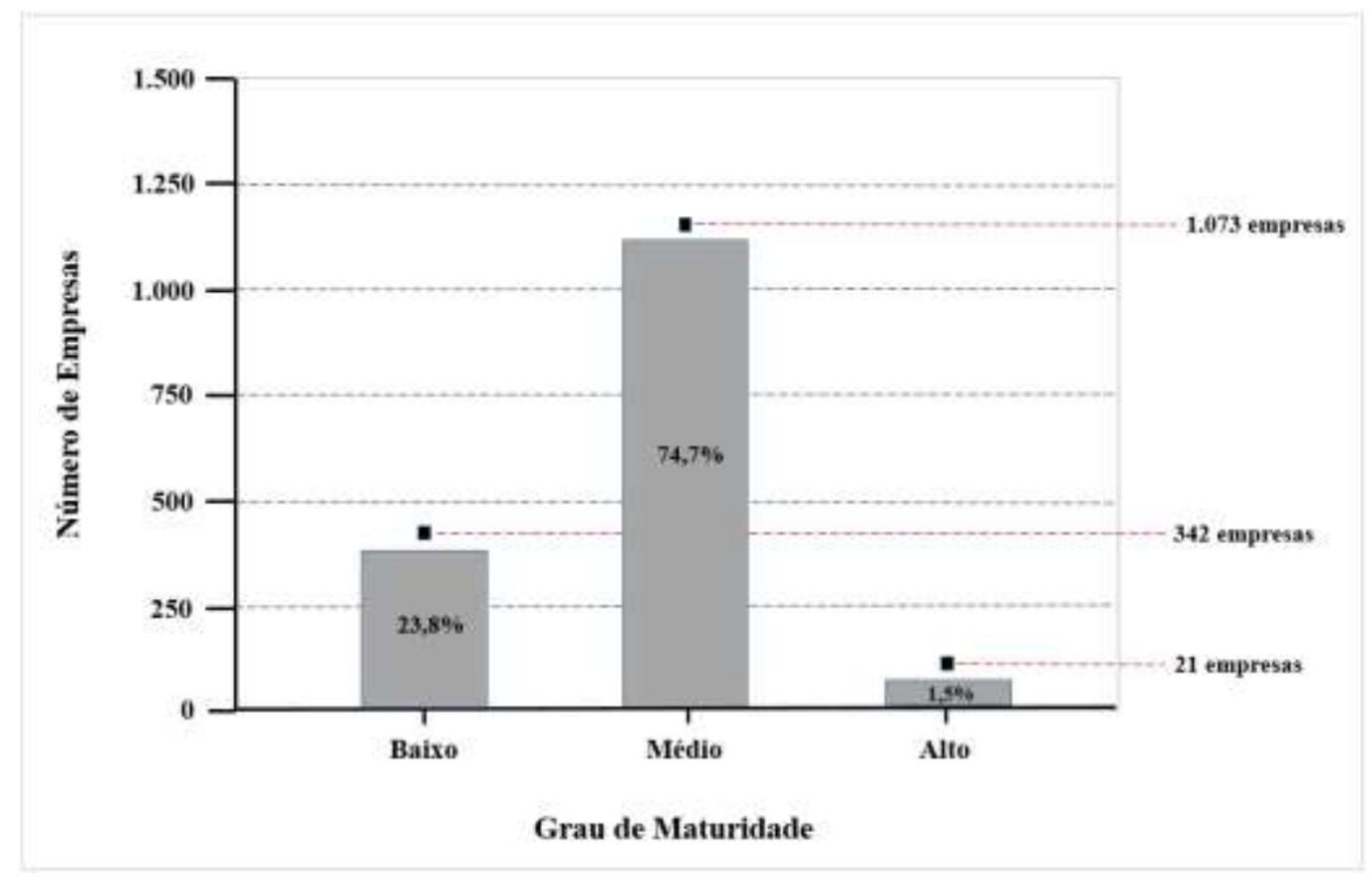

Figura 1. Grau de Maturidade Gerencial das Empresas Pecuaristas Brasileiras Fonte: Dados da pesquisa.

Empresas que apresentam grau médio de maturidade em gestão tecnológica (retomar Quadro 1) encaixam-se em um perfil que denota o uso de uma concepção gerencial para a condução das inovações tecnológicas, no caso a técnica da sexagem genética associada à inseminação artificial, dentro dos processos de produção. Embora a técnica represente melhorias na eficiência operacional, o gerenciamento da inovação tecnológica exige práticas de mensuração e controle (Harmon, 2004; Camargo et al., 2006; Ucar, 2018), o que demanda conhecimentos sobre a rentabilidade, como a contribuição da tecnologia ou da gestão inovadora no condicionamento da empresa à produtividade e aos ganhos econômicos.

As empresas pecuaristas que apresentaram grau médio, portanto, denotam maior esforço gerencial para a obtenção de uma produção sistematizada em função da aplicação de uma inovação tecnológica, que alcança sua efetividade gradativamente (Harmon, 2004; Ucar, 2018). Camargo et al. (2006) ressaltam que o uso contínuo e gradativo da genética bovina garante aprendizagem para os componentes da rede produtiva que envolve as técnicas de sexagem, desde as empresas de $\mathrm{P} \& \mathrm{D}$ até os pecuaristas.

Por outro lado, ferramentas de detalhamento e melhoramento da gestão de custos, como o Custo Operacional Efetivo (COE) e o Custo Operacional Total (COT), agregam valor para toda a cadeia produtiva agropecuária. Apesar disso, conforme exploram Alvarenga et al. (2014), a utilização do COE e do COT ainda representa um desafio para o agronegócio, devido ao uso 

pecuaristas

de outros métodos de custeio mais tradicionais (custo por absorção ou custo baseado em atividades).

O estabelecimento das empresas pecuaristas brasileiras em um nível intermediário de maturidade gerencial indica, assim, que o uso da técnica da sexagem associada à inseminação artificial não é fortuito, mas provavelmente planejado e acompanhado, desde a fase de implementação até a mensuração dos resultados, o que corrobora os resultados previamente apresentados por Camargo et al. (2004) e Camargo et al. (2006).

\section{Elementos de Convergência e Explicação para a Maturidade em Gestão Tecnológica}

Conhecendo-se o grau de maturidade em gestão tecnológica das empresas pecuaristas brasileiras usuárias de técnicas de genética bovina, visou-se levantar, em complemento, os elementos de convergência e explicação para tal constructo - a maturidade em gestão tecnológica. Desse modo, os itens foram submetidos a análise fatorial de componentes principais, fixada em um fator (ver seção 3.4), obtendo-se uma explicação de 25,60\% da variância total e cargas fatoriais que variaram de -0,088 a 0,751.

Uma vez que a análise de componentes principais teve fins de redução de itens e utilizou-se como critério de qualidade de explicação a significância prática - cargas fatoriais acima de 0,5 (Hair et al., 2010) -, os resultados evidenciaram 4 itens principais (dos 9 submetidos à análise) que explicam o componente 'Maturidade em Gestão Tecnológica' (Tabela 1).

\section{Tabela 1}

Itens principais que explicam o componente 'Maturidade em Gestão Tecnológica'

\begin{tabular}{l|c}
\hline Item & Carga Fatorial \\
\hline Mensuração da produtividade e de ganhos econômicos associados à gestão do negócio & 0,751 \\
\hline Rentabilidade advém da técnica e gestão & 0,680 \\
\hline Conhecimento sobre rentabilidade & 0,665 \\
\hline Adota COE e COT como ferramenta de gestão & 0,546 \\
\hline
\end{tabular}

Fonte: Dados da pesquisa.

Basicamente, os resultados da análise de componentes principais revelam os itens que melhor explicam o constructo da maturidade em gestão tecnológica. De tal modo, compreendese que a maturidade em gestão tecnológica, para as empresas pecuaristas brasileiras, é balizada (1) pela adoção de práticas de mensuração da produtividade e de ganhos econômicos em relação à gestão do negócio, (2) pela rentabilidade associada justamente ao emprego da tecnologia em sexagem genética e a uma gestão eficaz, (3) pelos conhecimentos sobre a rentabilidade do 
negócio e (4) pela adoção de ferramentas robustas de gestão de custos - especificamente o COE e o COT.

A explicação do constructo, por outro lado, pode ser também corroborada pela variação compartilhada entre os itens e o grau de maturidade em gestão tecnológica, evidenciando quais itens demonstram maior eficácia preditiva para a maturidade gerencial (Tabela 2).

\section{Tabela 2}

Correlação entre os itens e o Grau de Maturidade em Gestão Tecnológica

\begin{tabular}{lccc}
\hline \multirow{2}{*}{ Item } & \multicolumn{3}{c}{$\begin{array}{c}\text { Grau de Maturidade em } \\
\text { Gestão Tecnológica }\end{array}$} \\
\cline { 2 - 4 } & \multicolumn{1}{c}{$r$} & $p$ & $r^{2}$ \\
\hline Conhecimento sobre rentabilidade & 0,537 & 0,000 & 0,288 \\
Mensuração de produtividade e ganhos econômicos associados à gestão do & 0,504 & 0,000 & 0,254 \\
negócio & 0,433 & 0,000 & 0,187 \\
Rentabilidade advém de técnica e gestão & 0,265 & 0,000 & 0,070 \\
Adoção de COE e COT como ferramenta de gestão & & \\
\hline Fonte: Dados da pesquisa. Notas. A correlação é significativa no nível $p \leq 0,01$ & $\left(2\right.$ extremidades). $r^{2}$ designa o \\
coeficiente de determinação, que indica a proporção de variação em y (grau de maturidade) que partilha variação \\
com x (item).
\end{tabular}

Conforme a Tabela 2, todos os itens mostraram correlações significativas $(p \leq 0,01) \mathrm{em}$ relação ao grau de maturidade em gestão tecnológica. Apesar disso, o item que demonstrou maior relação preditiva à maturidade gerencial foi o conhecimento sobre rentabilidade $(r=$ 0,537; $p=0,000)$, denotando $28,8 \%$ da variação do grau de maturidade em gestão tecnológica $\left(r^{2}=0,288\right)$. Isso significa que, conhecer a rentabilidade do negócio - assim como práticas gerenciais de mensuração de produtividade e ganhos econômicos e de controle e análise de custos - pode refletir em uma variação positiva no grau de maturidade gerencial.

Por conseguinte, ao avaliar a opinião pessoal dos gestores das empresas pecuaristas quanto à tecnologia genética - independente da adoção de práticas de mensuração de produtividade e rentabilidade ou de controle de custos -, verifica-se que a maioria dos gestores indicou uma relação alta $(31,5 \%)$ ou muito alta $(37,6 \%)$ entre o aumento da produtividade e a tecnologia genética (Tabela 3). 

pecuaristas

\section{Tabela 3}

Tabulação cruzada entre Aumento na Produtividade com Tecnologia Genética e o Grau de Maturidade

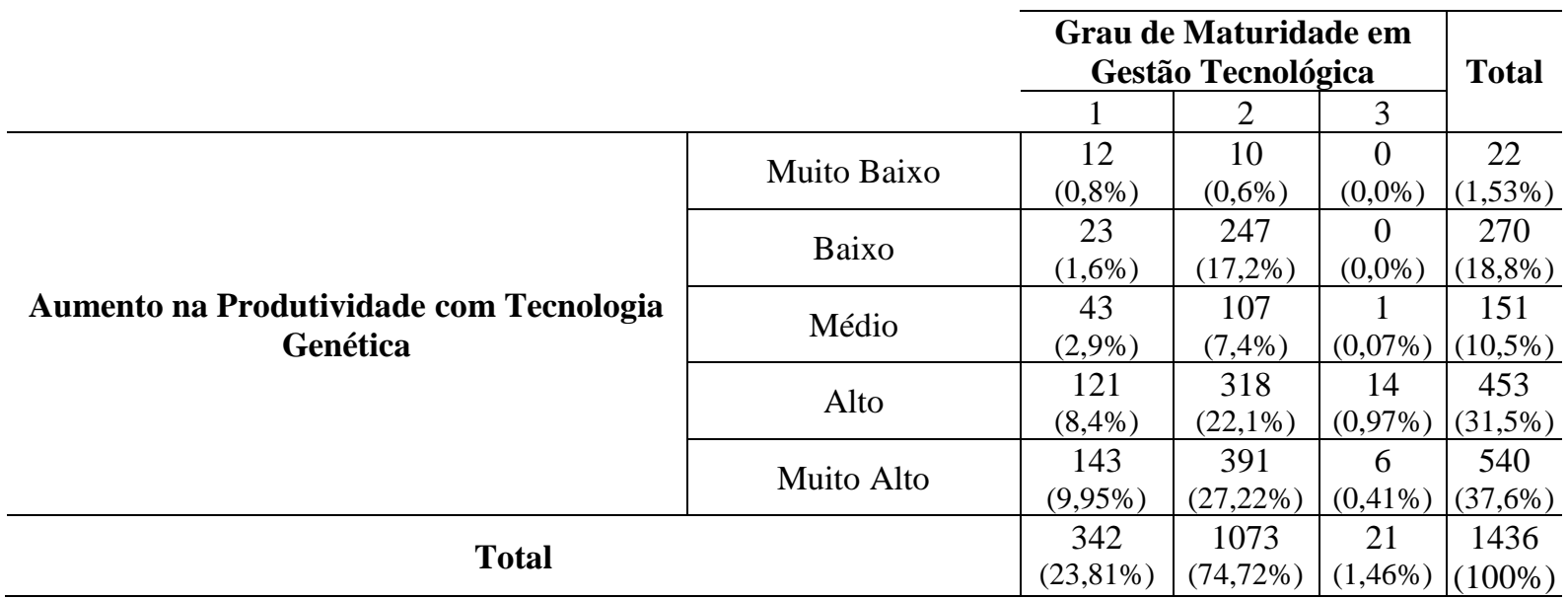

Fonte: Dados da Pesquisa. Notas. Em relação ao Grau de maturidade em Gestão Tecnológica, 1 significa Nível Baixo, 2 significa Nível Médio e 3 significa Nível Alto.

Além disso, com o uso do Coeficiente de Associação $\left(\chi^{2}\right)$ - utilizado para detecção de interação entre variáveis da amostra -, verificou-se que há a existência de relação entre a opinião dos gestores das empresas pecuaristas sobre a produtividade associada à tecnologia genética e o respectivo grau de maturidade em gestão tecnológica $\left[\chi^{2}(8)=68,033 ; p=0,000\right]$. A análise denota que, na medida em que as empresas pecuaristas encontram-se em níveis elevados de maturidade gerencial, o gestor tende a atribuir interdependência mais alta entre o aumento da produtividade e a tecnologia genética.

Os resultados encontrados corroboram, por exemplo, o que a literatura (Didonet \& Díaz, 2012; Maes \& Sels, 2014) pontua como fatores-chave para o sucesso de inovações tecnológicas no mercado: planejamento estratégico (metas e posicionamento) e gestão de custos (controle e decisões tomadas). De fato, tendo-se altos níveis de maturidade gerencial, é natural que a empresa reconheça os benefícios da inovação, uma vez que a proficuidade dos fatores gerenciais é garantida pela eficiência operacional inerente ao sistema produtivo da organização.

\section{Considerações Finais}

O estudo converge com a necessidade de compreender se as práticas de gerenciamento das empresas pecuaristas brasileras estão em consonância com as inovações tecnológicas do setor - o que sugere alto grau de maturidade gerencial -, uma vez que a otimização de custos e qualidade proporcionam produtividade e competitividade para essas empresas.

De tal modo, os resultados apontam um cenário interessante: a rentabilidade e os ganhos econômicos não dependem apenas da viabilidade técnica da tecnologia bovina, mas de uma 
série de co-fatores, como alinhamento estratégico, planejamento, adequação, controle e recursos. As empresas pecuaristas brasileiras, sobretudo por perfazerem uma larga cadeia produtiva, apresentam características cada vez mais interdependentes, bem como práticas gerenciais semelhantes, o que cria a necessidade de uma visão sistêmica, de modo que os aspectos funcionais do desenvolvimento possam ser alinhados às inovações tecnológicas.

Por outro lado, embora as empresas pecuaristas disponham de tecnologias de sexagem genética e fertilização in vitro, ambas capazes de preservar e acelarar ganhos genéticos ao produtor, são necessárias ações estratégicas, de modo que a tecnologia em si e seu respectivo uso não sejam os únicos relevantes. Sob o ponto de vista organizacional, no que compete à produtividade, competitividade e grau de aprendizagem, a sexagem genética e a fertilização in vitro cooperam positivamente para o aceleramento produtivo no setor pecuário. No entanto, sob o ponto contábil-financeiro, a vantagem competitiva não está apenas na tecnologia, mas no custo associado à economia de escala.

Essa constatação sugere que a maturidade gerencial das empresas pecuaristas seja sustentada pela cadeia produtiva, cuja integração e envolvimento são princípios para a efetiva difusão da sexagem genética e da fertilização in vitro, capaz de facilitar a gestão da inovação e o avanço tecnológico. Ou seja, relações mais estreitas estabelecidas e mantidas com os agentes institucionais da cadeia produtiva, como governo, mídia, universidades, instituições técnicas e associações comerciais são fundamentais para garantir maturidade gerencial no setor pecuário.

Diversas implicações gerenciais podem ser levantadas com este estudo. Basicamente, os resultados são contundentes em indicar que as empresas pecuaristas brasileiras são profícuas no uso das inovações tecnológicas em genética bovina. Ressalta-se, portanto, que a concepção de controle gerencial é de extrema importância para compreender os resultados aqui obtidos.

A principal limitação do estudo está na avaliação de elementos como produtividade e rentabilidade apenas considerando a visão subjetiva dos gestores das empresas pecuaristas investigadas. Isto é, relatórios gerenciais e contábeis trariam claramente resultados mais confiáveis e alinhados aos elementos avaliados. No entanto, considerando a dificuldade de acesso a relatórios gerenciais e contábeis e o tamanho da amostra utilizada (1.436 empresas), depreende-se que o estudo foi eficaz dentro das possibilidades operacionais estabelecidas.

Por fim, em função dos resultados obtidos e das limitações da pesquisa, sugerem-se estudos futuros que possam ampliar as unidades de análise, focando-se em informações 
financeiras e contábeis que representem produtividade e rentabilidade no setor pecuário, de forma que se possam investiga-los em profundidade.

\section{Referências}

Alkmin, D.V., Parrilla, I., Tarantini, T., \& Parlapan, L. 2014. Intra- and interboar variability in flow cytometric sperm sex sorting. Theriogenology, 82(1), 501-508.

Alvarenga, G.L., Castro Jr, L.G., Andrade, F.T., Costa, C.H.G., \& Oliveira, D.H. 2014. Metodologia de detalhamento e direcionamento da atuação no processo de gestão de custos da cafeicultura.Custos e @ gronegócio, 10(1),297-312.

Associação Brasileira de Inseminação Artificial - ASBIA. 2018. Available at: <www.asbia.org.br> Accessed 23 Jan. 2018.

Baruselli, P.S., Souza, A.H., Martins, C.M., Gimenes, L.U., Sales, J.N.S., Ayres, H., Andrade, A.F.C., Raphael, C.F., \& Arruda, R.P. 2007. Sêmen sexado: inseminação artificial e transferência de embriões. Revista Brasileira de Reprodução Animal, 31, 374-381.

Beltrame, R.T., Quirino, C.R., Barioni, L.G., \& Lima, V.F.M.H. 2010. Simulação e análise econômica da produção in vivo e in vitro de embriões em bovinos. Pesquisa Agropecuária Brasileira, 45(1), 1513-1520.

Burrus, R.T., Graham, J.E., \& Jones, A.T. 2018. Regional innovation and firm performance. Journal of Business Research, 88(1), 357-362.

Bylund, P.L., \& McCaffrey, M. 2017. A theory of entrepreneurship and institutional uncertainty. Journal of Business Venturing, 32(1), 461-475.

Camargo, S.H.C.R.V., Lima, N.C., Neves, M.F., Martinelli, D.P., \& Oliveira, M.M.B. 2004. Red Beef Connection e o canal de distribuição: Um estudo de caso na Chalet Agropecuária Ltda. Revista de Administração da Unimep, 2(1), 103-116.

Camargo, S.H.C.R.V., Lima, N.C., Martinelli, D.P., \& Oliveira, M.M.B. 2006. A capacitação tecnológica na pecuária bovina de corte: Um estudo de caso na Lagoa da Serra Ltda. Ciências Sociais em Perspectiva, 5(8), 85-101.

Cavalieri, F.L.B., Morotti, F., Seneda, M.M., Colombo, A.H.B., Andreazzi, M.A., Emanuelli, I.P., \& Rigolon, L.P. 2018. Improvement of bovine in vitro embryo production by ovarian follicular wave synchronization prior to ovum pick-up. Theriogenology, 117(1), 57-60.

Checa, M.L., Dunner, S., \& Cañón, J. 2002. Prediction of X and Y chromosome content in bovine sperm by using DNA pools through capillary electrophoresis. Theriogenology, 58(8), 1579-1586.

Clark, K.B., \& Fujimoto, T. 1991. Product development performance: strategy, organization and management in the world auto industry. Cambridge: Harvard Business School Press.

Cohen, R.J., Swerdlik, M.E., \& Sturman, E.D. 2014. Testagem e avaliação psicológica: introdução a testes e medidas. 8. ed. Porto Alegre: AMGH.

Empresa Brasileira de Pesquisa Agropecuária - EMBRAPA. 2018. Available at: <http://www.embrapa.br/>. Accessed 02 Mar. 2018.

Didonet, S.R., \& Díaz, G. 2012. Supply chain management practices as a support to innovation in SMEs. Journal of Technology Management \& Innovation, 7(3), 91-109. 
Fine C., \& Whitney, D. 1999. Is the make or buy decision a core competence? In: 4th International Symposium on Logistics (ISL'99), 1999, 4., Florence. Anais... Italy: ISL.

Freitas, C.P. 2007. Variações metodológicas na congelação de sêmen bovino sexado. Dissertação (Mestrado em Medicina Veterinária e Zootecnia) - Universidade Estadual Paulista "Júlio de Mesquita Filho" (UNESP), São Paulo.

Gao, Q.H., Wei, H.J., Luo, J., Han, C.M., Schoenian, S., Du, H.Z., Lu, Q.S., \& Qian, J. 2009. Flow cytometric sexing of X-and Y- chromosome-bearing sperm in Sika deer (Cervus nippon). Small Ruminant Research, 81(2-3), 100-104.

Garner, D.L. 2006. Flow cytometric sexing of mammalian sperm. Theriogenology, 65(5), $943-$ 957.

Garner, D.L., \& Seidel Jr., G.E. 2008. History of commercializing sexed semen for cattle. Theriogenology, 69(7), 886-895.

Hair, J.F., Black, W.C., Babin, B.J., Anderson, R.E., \& Tatham, R.L. 2010. Multivariate data analysis. Upper Saddle River: Pearson Education.

Harmon, P. 2004. Evaluating an organization's business process maturity. Business Process Trends, 2(3), 1-11.

Hall, J.B., Kasimanickam, R.K., Glaze Jr., J.B., \& Roberts-Lew, M.C. 2017. Impact of delayed insemination on pregnancy rates to gender selected semen in a fixed-time AI system. Theriogenology, 102(1), 154-161.

Hill, C.W.L., \& Rothaermel, F.T. 2003. The performance of incumbent firms in the face of radical technological innovation. Academy of Management Review, 28(2), 257-274.

Huang, Y., \& Chen, C. 2010. The impact of technological diversity and organizational slack on innovation. Technovation, 30(1), 420-428.

Judice, V.M.M., \& Baêta, A.M.C. 2005. Modelo empresarial, gestão de inovação e investimentos de venture capital em empresas de biotecnologia no Brasil. Revista de Administração Contemporânea, 9(1), 171-191.

Julca-Briceño, B.M., \& Neves, M.F. 2011. Caracterización de sistemas agroindustriales: Um estudio comparativo de los sectores sucroenergéticos de Brasil y Colombia. Interciencia, 36(1), 356-364.

Kostis, P.C., Kafka, K.I., \& Petrakis, P.E. 2018. Cultural change and innovation performance. Journal of Business Research, 88(1), 306-313.

Krishnan, V., \& Ulrich, K.T. 2001. Product development decisions: A review of the literature. Management Science, 47(1), 1-21.

Lima, V.F.M.H. 2007. Avanços metodológicos na seleção do sexo de espermatozóides bovinos para utilização no melhoramento genético e na produção animal. Revista Brasileira de Zootecnia, 36, 219-228.

Maes, J., \& Sels, L. 2014. SMEs' radical product innovation: The role of internally and externally oriented knowledge capabilities. Journal of Small Business Management, 52(1), 141-163.

Malhotra, N.K. 2011. Pesquisa de marketing: foco na decisão. $3^{\text {a }}$ ed., Pearson Prentice Hall, São Paulo. 
Matias-Pereira, J., \& Kruglianskas, I. 2005. Gestão de inovação: A lei de inovação tecnológica como ferramenta de apoio às políticas industrial e tecnológica do Brasil. RAE Eletrônica, $4(2), 1-21$.

Miguel, P.A.C., Carvalho, M.M., \& Lopes, A.P.V.B. 2013. A pilot case study of open innovation in a Brazilian company. Product: Management \& Development, 11(2), 136-141.

Nascimento, T. C., Veras, M., \& Milito, C.M. 2013. Maturidade em projetos públicos: Um estudo de caso do dimensionamento de seu gerenciamento. Sistemas \& Gestão, 8(3), 276288.

Nascimento, T.C., Veras, M., Milito, C.M., \& Oliveira Jr., P.C.M. 2014. Fatores que contribuem para a maturidade em gerenciamento de projetos: o caso de um governo estadual. Revista de Administração, 49(2), 415-428.

Parolin, S.R.H. 2013. Estudo multicasos sobre atividades inovativas. Revista de Administração, 48(3), 608-620.

Pellegrino, G., Piva, M., \& Vivarelli, M. 2012. Young firms and innovation: a microeconometric analysis. Structural Change and Economic Dynamics, 23(4), 329-340.

Pellegrino, C.A.G., Morotti, F., Untura, R.M., Pontes, J.H.F., Pellegrino, M.F.O., Campolina, J.P., Seneda, M.M. , Barbosa, F.A., \& Henry, M. 2016. Use of sexed sorted semen for fixedtime artificial insemination or fixed-time embryo transfer of in vitro-produced embryos in cattle. Theriogenology, 86(1), 888-893.

Penedo, A.S.T., Souza, G.H.S., Lima, N.C., Martins, E.S., Costa, A.C.S., Silva, T.E.E., \& Queiroz, J.V. 2014. Development of new products in a veterinary chemical company. International Journal of Economics, Commerce \& Management, 2(2), 1-11.

Ramos, A., \& Zilber, S.N. 2015. O impacto do investimento na capacidade inovadora da empresa. Revista de Administração e Inovação, 12(1), 303-325.

Rege, J.E.O., \& Gibson, J.P. 2003. Animal genetic resources and economic development: issues in relation to economic valuation. Ecological Economics, 45(3), 319-330.

Rege, J.E.O., Marshall, K., Notenbaert, A., Ojango, J.M.K., \& Okeyo, A.M. 2011. Pro-poor animal improvement and breeding - what can science do? Livestock Science, 136(1), 15-28.

Roud, V. 2018. Understanding the heterogeneity of innovation modes: Performance effects, barriers, and demand for state support. Technological Forecasting \& Social Change, 133(1), 238-253.

Seidel Jr., G.E. 2003. Economics of selecting for sex: the most important genetic trait. Theriogenology, 59(2), 585-598.

Seidel Jr, G.E. 2007. Overview of sexing sperm. Theriogenology, 68(3), 443-446.

Seidel Jr., G.E. 2009. Sperm sexing technology - The transition to commercial application: An introduction to the symposium "Update on sexing mammalian sperm". Theriogenology, 71(1), 1-3.

Sexing Technologies. 2016. Available at: <http://www.sexingtechnologies.com>. Accessed 02 Jan. 2016.

Sharpe, J.C., \& Evans, K.M. 2009. Advances in flow cytometry for sperm sexing. Theriogenology, 71(1), 4-10. 
Srinivasan, R., Lilien, G.L., \& Rangaswamy, A. 2006. The emergence of dominant designs. Journal of Marketing, 70(2), 1-17.

Suarez, F.F. 2004. Battles for technological dominance: an integrative framework. Research Policy, 33(1), 271-286.

Trigal, B., Gómez, E., Caamaño, J.N., Muñoz, M., Moreno, J., Carrocera, S., Martín, D., \& Diez, C. 2012. In vitro and in vivo quality of bovine embryos in vitro produced with sexsorted sperm. Theriogenology, 78(7), 1465-1475.

Ucar, E. 2018. Local creative culture and corporate innovation. Journal of Business Research, 91(1), 60-70.

Varago, F.C., Mendonça, L.F., \& Lagares, M.A. 2008. Produção in vitro de embriões bovinos: estado da arte e perspectivas de uma técnica em constante evolução. Revista Brasileira de Reprodução Animal, 32, 100-109.

Whetten, D.A. 2003. O que constitui uma contribuição teórica? Revista de Administração de Empresas, 43(3), 69-73.

Wignaraja, G. 2012. Innovation, learning, and exporting in China: does R\&D or a technology index matter? Journal of Asian Economics, 23(3), 224-233. 